\title{
Avaliação fitoquímica e toxicológica das folhas secas da Cynara scolymus
}

\author{
Phytochemical and toxicological evoluation of drt leaves of Cynara scolymus \\ Evaluación fitoquímica y toxicológica de hojas secas de Cynara scolymus
}

Recebido: 24/05/2021 | Revisado: 30/05/2021 | Aceito: 15/06/2021 | Publicado: 29/06/2021

\author{
Gleicy Kelly China Quemel \\ ORCID: https://orcid.org/0000-0003-1280-560X \\ Escola Superior da Amazônia, Brasil \\ E-mail: gkcquemel@gmail.com \\ Juan Gonzalo Bardalez Rivera \\ ORCID: https://orcid.org/0000-0003-1737-6947 \\ Escola Superior da Amazônia, Brasil \\ E-mail: jgrivera@bol.com.br \\ Ana Victoria de Brito Barbosa \\ ORCID: https://orcid.org/0000-0001-9534-0793 \\ Escola Superior da Amazônia, Brasil \\ E-mail: annafarma2017@gmail.com \\ Marianna Paiva Maciel \\ ORCID: https://orcid.org/0000-0002-1465-2160 \\ Escola Superior da Amazônia, Brasil \\ E-mail: mariannapaivamaciel@gmail.com \\ Gabriel dos Santos Moura \\ ORCID: https://orcid.org/0000-0002-7817-4292 \\ Escola Superior da Amazônia, Brasil \\ E-mail: htgabriel45@gmail.com
}

\begin{abstract}
Resumo
As indicações do uso de plantas medicinais para cura, prevenção e tratamento de doenças, desde a antiguidade, servem como importante fonte de informação para a busca de compostos com propriedades terapêuticas, a planta Cynara scolymus, conhecida como Alcachofra, apresenta-se como um vegetal de baixo valor calórico, rico em fibras e ferro, baixo teor de gordura, carboidratos, em sua maioria, formados pela inulina, é, indicado em dietas de diabéticos e pessoas com constipação intestinal, obesidade e também é recomendada como antianêmico. Diante dessas indicações terapêuticas este trabalho apresenta como objetivo a identificação do perfil fitoquímico no extrato hidroalccólico e análise toxicológica para avaliação de metemoglobina in vitro nos extratos hidroalcóolico e aquoso da alcachofra. A amostra para análise foi adquirida em um ervanário na Cidade de Belém, no estado do Pará, todas as análises foram feitas em triplicatas.Os resultados encontrados para análise fitoquímica qualitativa indicaram positivo para Alcaloides, Triterpenos, Taninos/Fenois e Saponinas, as demais identificações de metabolitos foram negativas; já os resultados de toxicidade de metamoglobina no extrato hidroalcolico toas as concentrações apresentaram porcentagens acima do permitido e no extrato aquoso, somente duas concentrações de $600 \mu \mathrm{g} / \mathrm{mL}$ e $500 \mu \mathrm{g} / \mathrm{mL}$, foram aceitáveis para o corpo humano. Conclui-se que a alcachofra apresenta atividade terapêutica, porém também apresentou um alto índice de porcentagem de toxicidade de metemoglobina.
\end{abstract}

Palavras-chave: Cynara scolymus; Metabólitos secundários; Toxicidade.

\begin{abstract}
Indications for the use of medicinal plants for the cure, prevention and treatment of diseases, since ancient times, serve as an important source of information for the search for compounds with therapeutic properties. Cynara scolymus plant, known as Artichoke, presents itself as a vegetable low in calories, rich in fiber and iron, low in fat, carbohydrates, mostly formed by inulin, it is indicated in diets for diabetics and people with constipation, obesity and is also recommended as an anti-anemic. Given these therapeutic indications, this work aims to identify the phytochemical profile in the hydroalcoholic extract and toxicological analysis for in vitro methemoglobin evaluation in the hydroalcoholic and aqueous artichoke extracts. The sample for analysis was acquired from an herbalist in the city of Belém, in the state of Pará, all analyzes were performed in triplicate. The results found for qualitative phytochemical analysis indicated positive for Alkaloids, Triterpenes, Tannins/Phenols and Saponins, the other identifications of metabolites were negative; on the other hand, the results of methemoglobin toxicity in the hydroalcoholic extract at all concentrations showed percentages above the permitted level, and in the aqueous extract, only two concentrations of $600 \mu \mathrm{g} / \mathrm{mL}$ and $500 \mu \mathrm{g} / \mathrm{mL}$ were acceptable for the human body. It is concluded that artichoke has therapeutic activity, but also presented a high percentage of methemoglobin toxicity.
\end{abstract}

Keywords: Cynara scolymus; Secondary metabolites; Toxicity. 


\begin{abstract}
Resumen
Las indicaciones para el uso de plantas medicinales para la cura, prevención y tratamiento de enfermedades, desde la antigüedad, sirven como una importante fuente de información para la búsqueda de compuestos con propiedades terapéuticas. La planta Cynara scolymus, conocida como Alcachofa, se presenta como un vegetal bajo en calorías, rico en fibra y hierro, bajo en grasas, carbohidratos, formado mayoritariamente por inulina, está indicado en dietas para diabéticos y personas con estreñimiento, obesidad y también se recomienda como antianémico. Dadas estas indicaciones terapéuticas, este trabajo tiene como objetivo identificar el perfil fitoquímico en el extracto hidroalcohólico y el análisis toxicológico para la evaluación in vitro de metahemoglobina en los extractos hidroalcohólicos y acuosos de alcachofa. La muestra para análisis se adquirió de un herbolario de la ciudad de Belém, en el estado de Pará, todos los análisis se realizaron por triplicado, los metabolitos resultaron negativos; por otro lado, los resultados de toxicidad de metahemoglobina en el extracto hidroalcohólico a todas las concentraciones mostraron porcentajes por encima del nivel permitido, y en el extracto acuoso solo dos concentraciones de $600 \mu \mathrm{g} / \mathrm{mL}$ y $500 \mu \mathrm{g}$ $/ \mathrm{mL}$ fueron aceptables para el cuerpo humano. Se concluye que la alcachofa tiene actividad terapéutica, pero también presenta un alto porcentaje de toxicidad por metahemoglobina.
\end{abstract}

Palabras clave: Cynara scolymus; Metabolitos secundários; Toxicidad.

\title{
1. Introdução
}

O uso de plantas medicinais é milenar, entretanto, desde o início deste século, tem ocorrido um crescente interesse pelo estudo de espécies vegetais e seu uso tradicional em diferentes partes do mundo. O conhecimento sobre seu cultivo e utilização simboliza, muitas vezes, o único recurso terapêutico de muitas comunidades e grupos étnicos (Araújo, Macena \& Campelo, 2017).

As plantas medicinais correspondem aos mais antigos hábitos de recorrer aos benefícios curativos dos vegetais, sendo que é uma das primeiras manifestações da humanidade para compreensão do tratamento de enfermidades. As observações populares sobre o uso e a eficácia de plantas medicinais, mantém em alta a prática do consumo de fitoterápicos, tornando válidas as informações terapêuticas que foram sendo acumuladas durante séculos (Zalewski, 2017).

No Brasil, a cultura de uso de plantas medicinais é presente em vários municípios, destacando-se a fitoterapia como uma opção medicamentosa no atendimento primário à saúde. De acordo com a OMS, diversos países, especialmente os que estão em desenvolvimento, já fazem o uso das plantas medicinais ou a formulação destas no tratamento de diversas doenças (Trindade et al., 2018).

A Alcachofra, é uma das mais antigas plantas medicinais cultivadas no mundo, chegou ao Brasil por intermédio dos imigrantes europeus, Por ser uma planta comestível, a Alcachofra é utilizada na cozinha brasileira, italiana e romana, que pode ser ser consumida refogada ou frita, com molhos ou em conserva. Todavia, além de ser considerada um prato saboroso, seu consumo vai muito além disso, especialmente, por ser rica em matérias minerais (Reolon-Costa et al, 2016).

É uma planta com ciclo vegetativo que dura por um tempo de dois anos, sendo considerada um vegetal vivaz e perene, pertencente à família das compostas. Cresce em forma de arbusto, até um metro de altura, com o caule marcado por estrias. As folhas, com discretos espinhos nos segmentos, são carnosas. Brácteas ou folhas modificadas, vermelhas ou verdes, desenvolvem-se umas sobre as outras, ao redor das flores azuis, formando capítulos compactos de até dez centímetros de diâmetro (Botsaris \& Alves, 2007).

Montanarini (2019), afirma que a alcachofra é considerada um importante estimulante do fígado, purificadora e fortificante do coração, assim como, pode dissolver cálculos renais, ser desintoxicante e agente sedativo da tosse. É composta, principalmente, de água e fibras, que auxiliam a regular o intestino, melhorar a digestão e a produção dos sucos gástricos/digestivos.

De acordo com a $1^{a}$ edição do memento fitoterápico, as indicações terapêuticas da alcachofra são antidispéptico, antiflatulento, diurético. Além de auxiliar na prevenção da aterosclerose e como coadjuvante no tratamento de dislipidemia mista leve a moderada e como auxiliar nos sintomas da síndrome do intestino irritável (Brasil, 2016) 
Com base nas propriedades farmacológicas já comprovadas da alcachofra, o trabalho tem como finalidade a prospecção fitoquímica no extrato hidroalcoólico a 70\% das folhas secas da alcachofra e a avaliação do potencial toxicológico nos extratos hidroalcoólico e aquoso das folhas secas da alcachofra.

\section{Metodologia}

A natureza da pesquisa foi aplicada com abordagem mista, pois apresentou características qualitativas e quantitativas (Gehardt \& Silveira, 2009). A amostra foi adquirida em ervanário na Cidade de Belém, no estado do Pará, o período da compra foi em dezembro de 2020. As folhas encontravam-se secas, na quantidade $1 \mathrm{~kg}$, acondicionadas em embalagem, lacrada e rotulada. Posteriormente foram levadas ao Laboratório de análises fitoquímicas e toxicológicas da Faculdade Escola Superior da Amazônia - ESAMAZ/Belém - PA para realização das análises, sendo que todas as análises foram feitas em triplicatas.

\subsection{Análises Fitoquímicas}

A determinação qualitativa dos marcadores químicos presentes na folha seca da Alcachofra foram utilizadas as metodologias de análise de Teixeira (2012), Paula e Lima (2019) e Simões et al. (2001) com adaptações.

Ácidos Orgânicos: Foi adicionado $5 \mathrm{~mL}$ de água destilada, filtrados, e em $2 \mathrm{~mL}$ do sobrenadante foi acrescido $500 \mu \mathrm{L}$ do reativo de Pascová. A descoloração do reativo indica a presença positiva de ácidos orgânicos na amostra.

Alcalóides: adicionou-se $2 \mathrm{~mL}$ de extrato hidroalcoólico, $1 \mathrm{~mL}$ ácido clorídrico (HCL) e 4 gotas de Bouchardt em um tubo de ensaio. A formação de precipitados insolúveis e floculoso confirmou a presença de alcalóides.

Antraquinonas: em um tubo de ensaio, foi adicionado $5 \mathrm{~mL}$ de extrato foliar da alcachofra e $5 \mathrm{~mL}$ de clorofórmio grau (P.A - ACS), agitou-se vigorosamente e deixado em repouso por 1 minuto. A fração orgânica foi recolhida e transferida para outro tubo de ensaio, em seguida foi acrescido $1 \mathrm{~mL}$ de solução aquosa de hidróxido de sódio $(\mathrm{NaOH}) 5 \%(\mathrm{~m} / \mathrm{v})$. A coloração roxa em fase aquosa indicou a presença de antraquinonas (reação de Bornträeger).

Cumarinas: em um papel filtro pingaram-se três gotas do extrato, em seguida, colocou-se três gotas de uma solução aquosa de hidróxido de sódio $(\mathrm{NaOH}) 1 \mathrm{M}$. Posteriormente, foi observado o aparecimento de fluorescência azul-brilhante ou verde sob a luz ultravioleta $(360 \mathrm{~nm})$.

Flavonoides: em $2 \mathrm{ml}$ do extrato foi adicionado $2 \mathrm{~mL}$ de ácido clorídrico $(\mathrm{HCl})$ e aproximadamente $0,5 \mathrm{~cm}$ de magnésio em fita com 2. O fim da reação se dá pelo término de efervescência. Aparecimento de coloração parda a vermelha indica a presença de flavonóides no extrato.

Purinas: em $2 \mathrm{~mL}$ de extrato aquoso foi adicionado 2 ácido clorídrico $(\mathrm{HCl})$. O aparecimento de coloração violeta indica reação positiva

Saponinas: em $2 \mathrm{ml}$ do extrato adicionou-se $2 \mathrm{ml}$ de água destilada e 3 gotas de ácido clorídrico (HCl), em seguida a solução agitar a solução por 3 minutos. A formação de espuma persistente e abundante indicou a presença de saponina.

Taninos: em $2 \mathrm{ml}$ do extrato foi acrescido três gotas de solução alcoólica de Cloreto de Ferro $\mathrm{III}\left(\mathrm{FeCl}_{3}\right)$, e posteriormente agitou-se vigorosamente.Precipitado de tonalidade azul indica a presença de taninos hidrolisáveis, e verde, a presença de taninos condensados.

Triterpenos: em $2 \mathrm{ml}$ do extrato foi adicionado a $2 \mathrm{ml}$ de clorofórmio, $1 \mathrm{ml}$ de anidrido acético e três gotas de ácido sulfúrico concentradp $\left(\mathrm{H}_{2} \mathrm{SO}_{4}\right)$ concentrado, posteriormente a solução foi agitada. A coloração azul evanescente seguida de verde, indicou a presença de triterpenóides respectivamente. 


\subsection{Análise Toxicológica em Metemoglobina}

O teste de toxicidade de metemoglobina usou dois tipos de extratos, hidroalcoólico $70 \%$ e Aquoso, onde foi usado a folha seca da Cynara scolymu, nas concentrações de: 1mg/mL, $900 \mu \mathrm{g} / \mathrm{mL}, 800 \mu \mathrm{g} / \mathrm{mL}, 700 \mu \mathrm{g} / \mathrm{mL}, 600 \mu \mathrm{g} / \mathrm{mL}$ e $500 \mu \mathrm{g} / \mathrm{mL}$, em amostra de sangue. O procedimento usou-se $0,5 \mathrm{ml}$ da amostra de sangue que foi centrifugado com soro fisiológico ate a superfície do tubo, esse processo foi realizado três vezes.

Foi transferido $500 \mu \mathrm{L}$ da amostra sanguínea e adicionado mais $100 \mu \mathrm{L}$ da solução mãe de extrato aquoso/hidroalcoólico $70 \%$, e deixado em repouso por 5 minutos. Usou-se $2,5 \mathrm{~mL}$ de água destilada em $0,5 \mathrm{~mL}$ de sedimento sanguíneo que estava em repouso com a solução mãe (extrato aquoso e hidroalcóolico 70\%), agitou-se lentamente três vezes e foi deixado em descanso por três minutos. Adicionou-se três gotas de Trinton X e $1 \mathrm{~mL}$ de tampão de fosfato, foi agitado no vortéx por 30 segundos, Adicionou-se em dois tubos, no tubo 1 (A1) havia $2,4 \mathrm{~mL}$ da amostra, no tubo 2 (A2) $0,2 \mathrm{~mL}$ da amostra mais $2,2 \mathrm{~mL}$ do ferrocianeto de fosfato. A leitura foi efetuada no espectrofotômetro, na absorbância de $632 \mathrm{~nm}$. Após a primeira leitura, foi adicionado $100 \mu \mathrm{L}$ de cianeto neutralizante, no tubo 1 (A3) e no tubo 2 (A4) levado para o vortéx por dez segundos, realizando novas leituras de absorbâncias em 632nm, no espectrofotômetro. Os resultados obtidos foram calculados utilizando a fórmula 1. Foram realizados testes paramétricos e não paramétricos, utilizando como programa estatístico o Bioestat 5.0.

Fórmula 1: Resultado das análises

$\% \mathrm{MeHb}=(\mathrm{A} 1-\mathrm{A} 3) \times 100$

$12 \mathrm{x}(\mathrm{A} 2-\mathrm{A} 4)$

Onde: $12=$ Fator de Diluição

$\mathrm{A} 1-\mathrm{A} 3=\%$ de metemoglobina na amostra;

A2-A4 $=100 \%$ de metemoglobina

\section{Resultados e Discussão}

$\mathrm{Na}$ análise dos metabólitos secundários é possível à identificação de substâncias que podem apresentar potencial efeito farmacológico, sendo que estes metabólitos são dependentes do ambiente que a planta se desenvolveu, os quais podem favorecer a presença ou ausência, bem como aumento ou diminuição na concentração desses metabólitos (Maciel et al., 2021). A Tabela 1 demonstra os resultados qualitativos dos testes fitoquímicos feitos no extrato hidroalcóolico a $70 \%$ da folha seca planta Cynara scolymus. 
Tabela 1: Resultados da prospecção fitoquímica (+ Positiva / - Negativa).

\begin{tabular}{cc}
\hline Testes & Resultados \\
\hline Alcalóides & + \\
\hline Ácidos Orgânicos & - \\
\hline Triterpenos & + \\
\hline Flavonóides & - \\
\hline Purinas & + \\
\hline Taninos & + \\
\hline Saponinas & - \\
\hline Cumarinas & - \\
\hline Antraquinonas &
\end{tabular}

Fonte: Autores (2021).

Na identificação de metabólitos secundários no extrato hidroalcóolico à 70\% das folhas secas apresentaram resultado positivo para alcaloides, triterpenos, taninos/fenóis e saponinas. De acordo com Costa et al., (2009) que avaliaram o extrato seco de alcachofra também não obtiveram resultado positivo para purinas, mas os espectros sugeriram a presença de flavonóides, cumarinas, antraquinonas e alcaloides.

De acordo com Monteiro et al., (2017) os principais metabólitos secundários encontrados na alcachofra foram a cinarina, o ácido clorogênico e o ácido caféico (derivados de compostos fenólicos), o lupeol (terpenos), a cinaropicrina (sesquiterpenos), o cinarosídeo e o escolimosídeo (flavonoides).

Nos estudos de Reolon-Costa et al., (2016) a parte da planta da alcachofra que possui interesse medicinal é a folha, e entre os diversos componentes químicos identificados destacam-se os flavonóides, os sesquiterpenos e os ácidos fenólicos.

Segundo Paula e Lima (2019), que avaliaram a prospecção fitoquímica de 37 amostras de chá de plantas medicinais para identificar a apresenta dos marcadores químicos responsáveis pelos efeitos terapêuticos, e para a alcachofra o marcador químico identificado foi o tanino, o que corrobora com os resultado semelhante ao resultado positivo presente no quadro 01 .

Quanto a presença positiva de saponinas nesse estudo são semelhantes aos de Oliveira et al., (2020) que revisaram pesquisas sobre os mecanismos de ação dos fitoterápicos e identificou que as saponinas tem ação anti-obesidade, as lactonas sesquiterpênicas tem ação antiespamôdica e ácidos fenólicos com ação anti-colerética.

Foi observado que existem divergências quanto à presença ou ausência de metabolitos secundários, podendo ocorrer, pois os componentes químicos presentes na Cynara scolymus são bem identificados em literaturas científicas, contudo, o cultivo da Alcachofra em diferentes regiões pode afetar a composição química da planta, seja por fatores sazonais ou ambientais (Monteiro et al, 2017)

A Norma Regulamentadora $n^{\circ} 7$ (NR-7) que trata do programa de controle de saúde ocupacional e dispõe dos parâmetros para o controle biológico da exposição ocupacional a alguns agentes químicos, preconiza o percentual de metemoglobina em $2 \%$, indicando que acima dessa porcentagem o indivíduo encontra-se potencialmente intoxicado. (Maciel et al., 2021).

A Tabela 2 apresenta os valores da média e do desvio-padrão das análises da determinação dos percentuais de metemoglobina das amostras do extrato hidroalcóolicoa 70\% e aquoso. Foi observado que há um aumento nos valores da média e do desvio padrão no extrato hidroalcóolico $(10,97 \pm 3,54)$ do que nas amostras aquosas $(4,89 \pm 3,93)$. 
Tabela 2: Resultado das Análises Toxicológicas.

\begin{tabular}{c|c|c|c}
\hline Pontos de concentração & $\mathrm{n}=3$ & $\begin{array}{c}\text { Folha } \\
\text { (Extrato hidroalcoólico 70\%) }\end{array}$ & $\begin{array}{c}\text { Folha } \\
\text { (Extrato aquoso) }\end{array}$ \\
\hline $1 \mathrm{mg} / \mathrm{mL}$ & 3 & $16,6 \%$ & $10,42 \%$ \\
\hline $900 \mu \mathrm{g} / \mathrm{mL}$ & 3 & $13,42 \%$ & $8,33 \%$ \\
\hline $800 \mu \mathrm{g} / \mathrm{mL}$ & 3 & $11,05 \%$ & $5,24 \%$ \\
\hline $700 \mu \mathrm{g} / \mathrm{mL}$ & 3 & $9,65 \%$ & $3,78 \%$ \\
\hline $600 \mu \mathrm{g} / \mathrm{mL}$ & 3 & $7,92 \%$ & $1,15 \%$ \\
\hline $500 \mu \mathrm{g} / \mathrm{mL}$ & 3 & $7,22 \%$ & $0,47 \%$ \\
\hline $\mathrm{X}$ & & 10,97 & 4,89 \\
\hline $\mathrm{Dp}$ & & 3,54 & 3,93 \\
\hline
\end{tabular}

Fonte: Autores (2021).

Já no Gráfico 1, é representado pela análise entre as médias do \% metemoglobina entre o extrato hidroalcoólico e aquoso de Cynara scolymus. Nela, podemos constatar que, quando analisado entre as concentrações estudadas para cada extrato, observamos que para o extrato hidroalcoólico, o valor foi de $p<0,01$ e para o extrato aquoso também e de $p<0,01$, sendo as análises significativas. Porém, quando analisado a diferenças entre as médias do \% de metemoglobina para cada extrato, não é visto significância.

Gráfico 1: Análise entre as médias do \% metemoglobina entre o extrato hidroalcoólico e aquoso.

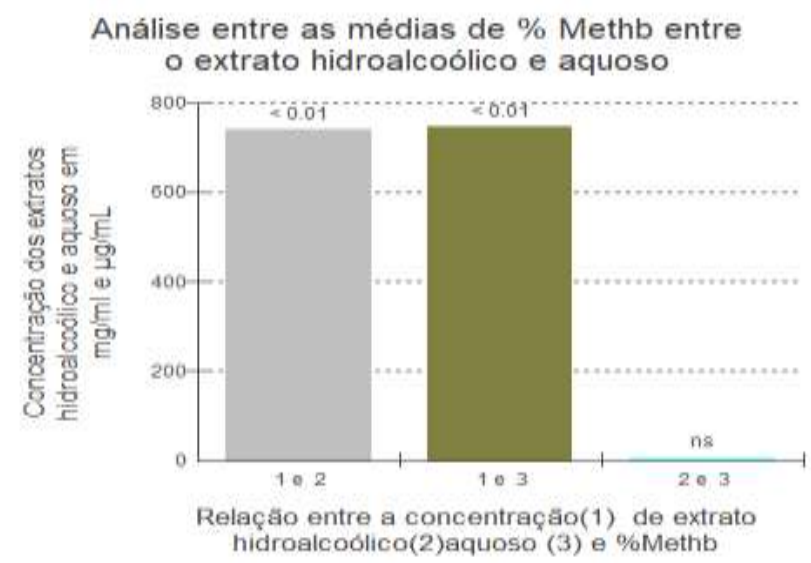

Fonte: Autores (2021).

Os Gráficos 2 e 3, mostram a correlação de Pearson realizada entre as concentrações estudadas e os extratos hidroalcóolico e aquoso da folhas secas da alcachofra. Foi observado que o valor de p foi significativo tanto para extrato hidroalcóolico $(\mathrm{p}=0,0008)$ quanto para o extrato aquoso $(\mathrm{p}=0.0002)$, pois ambos apresentam valor $\mathrm{p}<0.05$. 
Gráfico 2: Correlação de Pearson da \% de metemoglobina entre as concentrações e o extrato hidroalóolico a 70\% das folhas secas de alcachofra.

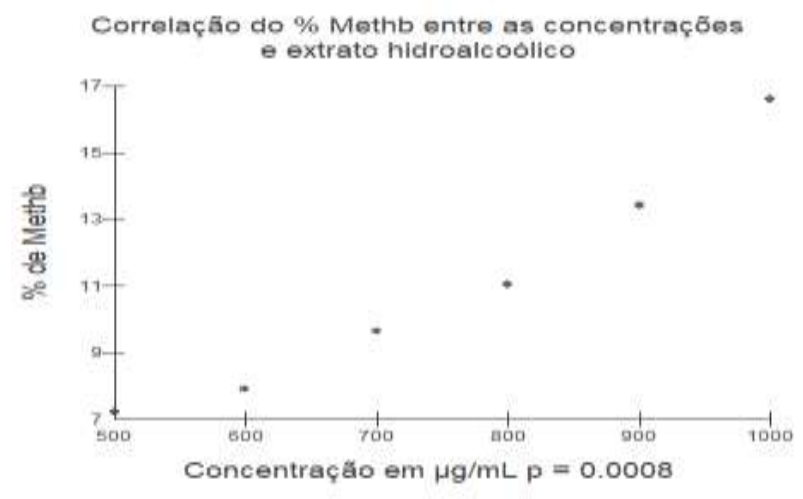

Fonte: Autores (2021).

Gráfico 3: Correlação de Pearson da \% de metemoglobina entre as concentrações e o extrato aquoso folhas secas de alcachofra.

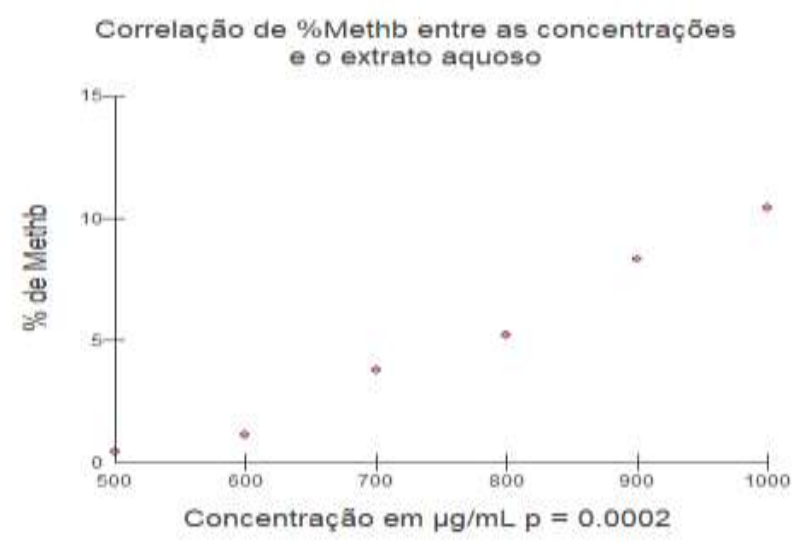

Fonte: Autores (2021).

Os diversos estudos realizados com a Alcachofra (Cynara scolymus) são capazes de comprovar as várias contribuições terapêuticas no âmbito medicinal. A formulação de um fitoterápico a partir desta planta pode auxiliar no tratamento de algumas patologias sendo utilizada em conjunto ou até substituindo um medicamento alopático.

Segundo estudos de Di-Stasi (1996), as preparações de folhas de alcachofra mostraram boa tolerabilidade e um baixo grau de efeitos colaterais, o que confirma a segurança desta droga. Contudo, por vezes, reações alérgicas foram relatadas após administração oral (rinite alérgica, asma brônquica, urticária), assim, o uso com cautela é recomendado em caso de alergia.

Estudo feito por Shimoda et al. (2003) mostraram que, o extrato de folha de alcachofra tem sido sugerido também para o tratamento de icterícia e insuficiência hepática. Dentre os componentes químicos presentes nesta planta encontram-se a cinarina, ácido caféico, ácido clorogênico e luteolina, responsáveis por inibir o estresse oxidativo em humanos.

Já no estudo de Artner-Dworzak et al., (2000) observou que, a alcachofra pode ser utilizada no tratamento contra hipercolesterolemia, no qual foi visto diminuição acentuada do colesterol sérico. Isto é justificado, por meio de análises quantitativas feitas com o extrato de alcachofra que inibe a biossíntese do colesterol de maneira dependente da concentração.

No estudo de Gebhardt, Rexhepaj e Fausel (1999) constataram que a Cinarina (ácido 1,5-dicafeilquínico) é o principal componente ativo da alcachofra. Somado a esse resultado, o flavonóide luteolina apresentam efeitos inibitórios sobre a síntese de colesterol. Além disso, observaram que, o extrato de alcachofra possui propriedades antioxidantes. Neste sentido, 
verificaram que o extrato protegeu culturas de hepatócitos de rato contra hidroperóxido produzido pelo estresse oxidativo. Outro dado foi que alcachofra inibiu também a oxidação do LDL e reduziu a produção intracelular de espécies reativas de oxigênio pelo LDL oxidado em culturas de células endoteliais e monócitos.

Jimenes-Escrig et al. (2003) relataram que, a planta possui atividade antioxidante, comparável à atividade da vitamina C e da vitamina E, e ainda é eficaz na inibição da oxidação das lipoproteínas de baixa densidade in vitro.

Porém, o uso tradicional de um remédio à base de plantas implica em relativa segurança, mas não garante que esses recursos estão livres eventos adversos e ou tóxicos (Ernst, 1998).

No estudo feito por Held (1992) indicou a ausência de eventos adversos graves em pacientes tratados com 1,8-1,9 g diárias de alcachofra. Foi visto no estudo que, dos 417 avaliados, $95 \%$ do pacientes apresentaram uma boa e excelente tolerabilidade para o extrato de alcachofra.

Fintelmann (1999) demonstrou no seu estudo de pós-comercialização feito em 203 pacientes, durante um período de 23 semanas, a ausência de eventos adversos e ou tóxicos.

Foram localizados poucos ou quase nada de ensaios clínicos que investigaram os efeitos metemoglobinizantes do extrato da alcachofra em modelos in vitro, que demonstrem os feitos tóxicos da mesma, no sistema sanguíneo.

\section{Conclusão}

Diante dos resultados apresentados nas análises fitoquímicas concluímos que a folha da planta Cynara scolymus, apresenta metabolitos secundários que estão diretamente ligados a sua atividade terapêutica, podendo ser utilizado em alguns tratamentos terapêuticos com o intuito de demonstrar possibilidades de terapias alternativas voltadas a fitoterápicos e promover o bem estar de adeptos a utilizadores de plantas medicinais, contudo, as análises toxicológicas de metemoglobina apresentou um resultado significativo de que a folha da planta apresenta uma considerável porcentagem de toxicidade, pois os resultados apontam que as concentrações do extrato hidroalcoolico foram todas com porcentagens acima do valor permitido pela norma regulamentadora 7, já o extrato aquoso demonstrou que a utilização em baixas concentrações como $600 \mu \mathrm{g} / \mathrm{mL}$ e $500 \mu \mathrm{g} / \mathrm{mL}$, torna-se aceitável para o corpo humano.

Visto que os resultados foram satisfatórios quanto à atividade terapêutica da planta, este trabalho endossa que ainda existe varias descobertas a serem feitas voltadas aos benefícios terapêutico da Cynara scolymus, e que a planta precisa cada vez ser mais estudada, com o intuito de no futuro ser produzido um fármaco eficaz e seletivo.

\section{Referências}

Araújo, R. J. B. L., Macena, I.D. \& Campelo, M.C.S. (2017) Hibiscus sabdariffa L. e seus benefícios funcionais a saúde humana com ênfase no Chá de Hibiscos. In: II Congresso Brasileiro de Ciências da Saúde. https://editorarealize.com.br/editora/anais/conbracis/2017/TRAB ALHO_EV071_MD4_SA6_ID1650_02052017210324.pdf

Artner-Dworzak, E., Mayr, O., Mueller, B., Maly, K. \& Grunicke, H. (2000). Influence of the artichoke extract on lipid metabolism. Phytomedicine; S2:46 SL91.

Botsaris, A. S. \& Alves, L. F. (2007) Cynara scolymus L. (Alcachofra). Revista Fitos, 2(4): 51 - 63

Brasil. Agência Nacional de Vigilância Sanitária. (2016). Memento Fitoterápico: Farmacopéia Brasileira. http://www.farmacia.pe.gov.br/sites/farmacia.saude.pe.gov.br/files/memento_fitoterapico.pdf

Costa, R. S., Ozela, E. F., Barbosa, W. L. R., Pereira, N. L. \& Silva Junior, J. O. C. (2009). Caracterização física, química e físico-química do extrato seco por nebulização (spray-drying) de Cynara scolmus L. (Asteraceae). Revista Brasileira de Farmacognosia, 90, $169-174$.

Di-stasi, L.C. (1996). Plantas medicinais: Arte e ciência. Um guia de estudo interdisciplinar. Ed. Univ. Estadual Paulista, São Paulo, Brasil, pp 230, 1996

Ernst, E., De-Smet, P. A., Shaw, D. \& Murray, V (1998). Traditional remedies and the test of time. Eur J Clin Pharmacol. 54:99-100.

Fintelmann, V. (1999). Artischockenextrakt bei dyspeptischem Symptomenkomplex. Methodik und Ergebnisse einer Anwendungsbeobachtung. Zeitschrift fuer Phytotherapie. 20:93-95. 
Gebhardt, R., Rexhepaj, R. \& Fausel, M. (1999). Antioxidative and hepatoprotective effect of flavonoids from artichoke leaf extracts. Z Phytother. 20(2):97-8.

Gerhardt, T. E. \& Silveira, D. T.[org]. (2009) Métodos de Pesquisa. Editora da UFRGS. 120 p.

Held, C. (1992). Von der deutsch-ungarischen Phytopharmakakonferenz. Z Klin Med; 47:92.

Jiménez-Escrig, A., Dragsted, L. O., Daneshvar. B., Pulido. R. \& Saura-Calixto, F. (2003). In vitro antioxidant activities of edible artichoke (Cynara scolymus L.) and effect on biomarkers of antioxidants in rats. J Agric Food Chem 27;51(18):5540-5. 10.1021/jf030047e.

Maciel, M. P, Tavares, M. L., Quemel, G. K. C. \& Rivera, J. (2021). Análises físicas, bromatologicas, fitoquímicas e toxicológicas do fruto da planta Physalis angulata Lin. International Journal of Development Research. 11, 45488-45493 https://doi.org/10.37118/ijdr.21427.03.2021.

Monteiro, I. G. Pereira, I. R. S., Teixeira, J. J. M, Beserra, S. J. O. \& Silva, J. S. S. (2019) Atividade terapêutica e composição química da alcachofra (Cynara Scolymus Linné). Uma revisão da literatura. In: Anais da Mostra de Pesquisa em Ciência e Tecnologia Anais.Fortaleza(CE) DeVry Brasil - Damásio - Ibmec. https://www.even3.com.br/anais/mpct2017/47805-atividade-terapeutica-e-composicao-quimica-da-alcachofra-(cynara-scolymus-linne)-uma-revisao-daliteratura/

Paula, A. G. P. \& Lima, C. P. (2019). Prospecção fitoquímica e interações medicamentosas do chá de 37 ervas comercializado em Pinhais-PR. Brazilian Journal of health Review. 2, 4621-4640 10.34119/bjhrv2n5-063.

Reolon-Costa, A. Grando, M. \& Cravero, V. (2016) Alcachofra: (cynara cardunculus L. var scolymus L. Fiori): alimento funcional e fonte de compostos promotores da saúde. Revista Fitos. 10(4): 375 - 547.

Shimoda, H., Ninomiya, K., Nishida, N., Yoshina, T., Morikawa, T., Matsuda, H. \& Yoshida, M. (2003).Anti-hyperlipidemic sesquiterpenes and new sesquiterpene glycosides from the leaves of artichoke (Cynara scolymus L.): structure requirement and mode of actionBioorg. Med. Chem. Lett. 13:223-228.

Simões, C. M. O., Schenkel, E. P., Gosmann, G., Mello, J. C. P., Mentz, L. A. \& Petrovick, P. R. (org.) (2001). Farmacognosia: da planta ao medicamento. (3a ed.), Editora da Universidade UFRGS/ Editora da UFSC. 1104f.

Teixeira, L. N., Lima, M. A., Silva, M. M. \& Carvalho, L. F. M. (2021) Fitoquímico e avaliação do potencial de captura do radical dpph pelos extratos de Manilkara sapota L. In: VIICONNEPI Congresso Norte Nordeste de Pesquisa e Inovação. Palmas - Tocantins. 2012. https://propi.ifto.edu.br/ocs/index.php/connepi/vii/paper/view/1542

Zalewski, S., Silva, F. T. O., Jesus, V. C. \& Martines, K. N. M (2017). Levantamento de dados da população de Araraquara acerca do uso de plantas medicinais e o conhecimento sobre medicamentos fitoterápicos na XIX semana de assistência farmacêutica estudantil. Revista de Ciências Farmacêuticas Básica e Aplicada, 38(1): 1 - 10 